\title{
An Exact Series Solution for the Vibration of Mindlin Rectangular Plates with Elastically Restrained Edges
}

\author{
Xue Kai, Wang Jiufa, Li Qiuhong, Wang Weiyuan, and Wang Ping \\ College of Mechanical and Electrical Engineering, Harbin Engineering University, Harbin 150001, China \\ Correspondence should be addressed to Wang Jiufa; wangjiufa1987@sina.com
}

Received 4 March 2013; Accepted 19 September 2013; Published 25 February 2014

Academic Editor: Nuno Maia

Copyright ( 2014 Xue Kai et al. This is an open access article distributed under the Creative Commons Attribution License, which permits unrestricted use, distribution, and reproduction in any medium, provided the original work is properly cited.

\begin{abstract}
An analysis method is proposed for the vibration analysis of the Mindlin rectangular plates with general elastically restrained edges, in which the vibration displacements and the cross-sectional rotations of the mid-plane are expressed as the linear combination of a double Fourier cosine series and four one-dimensional Fourier series. The use of these supplementary functions is to solve the possible discontinuities with first derivatives at each edge. So this method can be applied to get the exact solution for vibration of plates with general elastic boundary conditions. The matrix eigenvalue equation which is equivalent to governing differential equations of the plate can be derived through using the boundary conditions and the governing equations based on Mindlin plate theory. The natural frequencies can be got through solving the matrix equation. Finally the numerical results are presented to validate the accuracy of the method.
\end{abstract}

\section{Introduction}

Rectangular plates are important structural elements and the analysis of the vibration is very important for the design of plate-type structures in aerospace, electronic, mechanical, marine, nuclear, and structural engineering. Thus, many researchers have done much work and got a lot of results. However, this research is based on the classical Kirchhoff hypothesis. This theory neglects the effect of shear deformation and rotary inertia which result in the over-estimation of vibration frequencies. This deviation will increase with increasing plate thickness. To improve the results, the Mindlin first-order plate can be employed. So the vibration of Mindlin rectangular plates has begun to gain attention.

The effective methods used to analyze the vibration of Mindlin rectangular plates include Rayleigh-Ritz method [1-4] and some numerical methods, such as differential quadrature method (DQM) $[5,6]$ and discrete singular convolution method (DSC) $[7,8]$. Several analysis methods were also proposed by some researchers. Hashemi et al. derived the exact close form characteristic equations and their associated eigenfunctions for the thick rectangular plates with two opposite sides simply supported $[9,10]$. Gorman used the superposition method to obtain a solution for the Mindlin plates [11, 12].

Most existing studies are limited to the classical homogeneous boundary conditions. Recently, Li proposed a Fourier series method for the vibration analysis of arbitrarily supported beam [13]. The flexural displacement of the beam is sought as the linear combination of a Fourier series and an auxiliary polynomial function. Subsequently, this method is extended to the flexural and in-plane vibration of rectangular plates under general boundary conditions. The flexural and in-plane displacement of the plate is sought as the linear combination of a double Fourier cosine series and auxiliary series functions [14-16]. It has been shown that this solution method works very well for various edge supports.

In this paper, an improved Fourier series method is employed to analyze the free vibration of Mindlin rectangular plates with general elastic boundary supports, in which the vibration displacements and the cross-sectional rotations of the mid-plane are expressed as the linear combination of a double Fourier cosine series and four one-dimensional Fourier series. The possible discontinuities problems, which maybe encountered in the displacement and rotations partial differentials along the edges, can be solved by these 


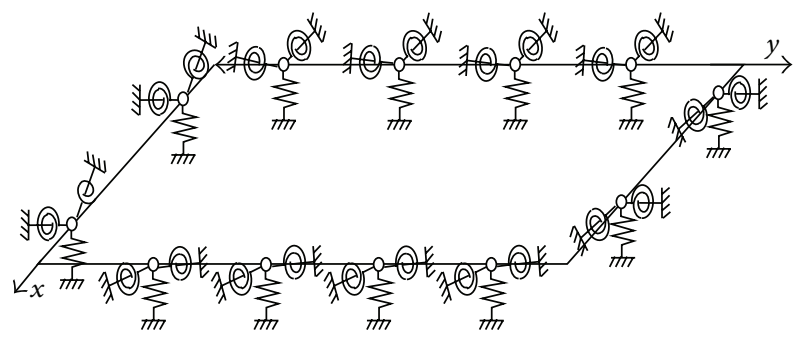

Figure 1: A Mindlin plate with general elastic boundary support.

supplementary functions. Then, an exact solution for Mindlin rectangular plates with arbitrary elastically restrained edges can be obtained. Finally several numerical examples and the comparisons with those results reported in the literature are presented to validate the accuracy of the present approach.

\section{Mathematical Modeling and Solution Methodology}

Consider a rectangular Mindlin plate elastically restrained along all edges, as shown in Figure 1. The boundary conditions are physically realized in terms of three kinds of restraining springs (translational, rotational, and torsional springs) attached to each edge. Different boundary conditions can be directly obtained by changing the stiffness of springs. The governing differential equations for free vibration of a Mindlin plate are given by

$$
\begin{gathered}
k G h\left(\frac{\partial^{2} w}{\partial x^{2}}+\frac{\partial^{2} w}{\partial y^{2}}+\frac{\partial \psi_{x}}{\partial x}+\frac{\partial \psi_{y}}{\partial y}\right)-\rho h \frac{\partial^{2} w}{\partial t^{2}}=0 \\
D\left(\frac{\partial^{2} \psi_{x}}{\partial x^{2}}+\frac{1-\mu}{2} \frac{\partial^{2} \psi_{x}}{\partial y^{2}}+\frac{1+\mu}{2} \frac{\partial^{2} \psi_{y}}{\partial x \partial y}\right) \\
-k G h\left(\frac{\partial w}{\partial x}+\psi_{x}\right)-\rho h \frac{\partial^{2} \psi_{x}}{\partial t^{2}}=0 \\
D\left(\frac{\partial^{2} \psi_{y}}{\partial y^{2}}+\frac{1-\mu}{2} \frac{\partial^{2} \psi_{y}}{\partial x^{2}}+\frac{1+\mu}{2} \frac{\partial^{2} \psi_{x}}{\partial x \partial y}\right) \\
-k G h\left(\frac{\partial w}{\partial y}+\psi_{y}\right)-\rho h \frac{\partial^{2} \psi_{y}}{\partial t^{2}}=0
\end{gathered}
$$

where $w$ is the transverse displacement, $\psi_{x}$ and $\psi_{y}$ are the slope due to bending alone in the respective planes, $k$ is the shear correction factor to account for the fact, $G=E / 2(1+\mu)$ is the shear modulus, $\mu$ is the Poisson's ratio, $D=E h^{3} /(12(1-$ $\left.\mu^{2}\right)$ ) is the flexural rigidity, $\rho$ is the mass density, and $h$ is the thickness of the plate.

In terms of transverse displacements and slope, the bending and twisting moments and the transverse shearing forces in plates can be expressed as

$$
\begin{aligned}
& M_{x}=D\left(\frac{\partial \psi_{x}}{\partial x}+\mu \frac{\partial \psi_{y}}{\partial y}\right), \\
& M_{y}=D\left(\frac{\partial \psi_{y}}{\partial y}+\mu \frac{\partial \psi_{x}}{\partial x}\right),
\end{aligned}
$$

$$
\begin{gathered}
M_{x y}=\frac{1-\mu}{2} D\left(\frac{\partial \psi_{x}}{\partial y}+\frac{\partial \psi_{y}}{\partial x}\right), \\
Q_{y}=k G h\left(\frac{\partial w}{\partial y}+\psi_{y}\right), \\
Q_{x}=k G h\left(\frac{\partial w}{\partial x}+\psi_{x}\right) .
\end{gathered}
$$

There are three forces along every edge and they are the bending moment, the twisting moment, and the shearing forces. Three kinds of restraining springs (rotational, torsional, and translational springs) along every edge can be corresponding to these three forces. The boundary conditions for an elastically restrained rectangular plate are as follows:

$$
\begin{gathered}
k_{x 0} w=-Q_{x}, \\
K_{x 0} \psi_{x}=-M_{x}, \\
K_{y x 0} \psi_{y}=-M_{x y}, \\
\text { at } x=0 ; \\
k_{x a} w=Q_{x}, \\
K_{x a} \psi_{x}=M_{x}, \\
K_{y x a} \psi_{y}=M_{x y}, \\
\text { at } x=a ; \\
k_{y 0} w=-Q_{y}, \\
K_{y 0} \psi_{y}=-M_{y}, \\
K_{x y 0} \psi_{x}=-M_{x y}, \\
\text { at } y=0 ; \\
k_{y b} w=Q_{y}, \\
K_{y b} \psi_{y}=M_{y}, \\
K_{x y b} \psi_{x}=M_{x y}, \\
\text { at } y=b,
\end{gathered}
$$

where $k_{x 0}$ and $k_{x a}\left(k_{y 0}\right.$ and $\left.k_{y b}\right)$ are the translation spring constants, $K_{x 0}$ and $K_{x a}\left(K_{y 0}\right.$ and $\left.K_{y b}\right)$ are the rotational spring constants, and $K_{y x 0}$ and $K_{y x a}\left(K_{x y 0}\right.$ and $\left.K_{x y b}\right)$ are the torsional spring constants at $x=0$ and $x=a(y=0$ and $y=b$ ), respectively. All classical homogeneous boundary conditions can be easily derived by simply setting each of the spring constants to be infinite or zero.

According to the Mindlin plate theory, the transverse displacement of the plate median surface and the rotations of the cross-section, respectively, along the $x$ direction and the $y$ direction are utilized. In this study, these quantities are expressed in form of improved Fourier series expansions [16]:

$$
\begin{aligned}
& w(x, y) \\
& =\sum_{m=0}^{\infty} \sum_{n=0}^{\infty} A_{m n} \cos \left(\lambda_{m} x\right) \cos \left(\lambda_{n} y\right) \\
& \quad+\sum_{l=1}^{2}\left(\xi_{l b}(y) \sum_{m=0}^{\infty} d_{l m}^{1} \cos \left(\lambda_{m} x\right)+\xi_{l a}(x) \sum_{n=0}^{\infty} f_{l n}^{1} \cos \left(\lambda_{n} y\right)\right),
\end{aligned}
$$




$$
\begin{aligned}
& \psi_{x}(x, y) \\
& =\sum_{m=0}^{\infty} \sum_{n=0}^{\infty} B_{m n} \cos \left(\lambda_{m} x\right) \cos \left(\lambda_{n} y\right) \\
& \quad+\sum_{l=1}^{2}\left(\xi_{l b}(y) \sum_{m=0}^{\infty} d_{l m}^{2} \cos \left(\lambda_{m} x\right)+\xi_{l a}(x) \sum_{n=0}^{\infty} f_{l n}^{2} \cos \left(\lambda_{n} y\right)\right), \\
& \psi_{y}(x, y) \\
& =\sum_{m=0}^{\infty} \sum_{n=0}^{\infty} C_{m n} \cos \left(\lambda_{m} x\right) \cos \left(\lambda_{n} y\right) \\
& \quad+\sum_{l=1}^{2}\left(\xi_{l b}(y) \sum_{m=0}^{\infty} d_{l m}^{3} \cos \left(\lambda_{m} x\right)+\xi_{l a}(x) \sum_{n=0}^{\infty} f_{l n}^{3} \cos \left(\lambda_{n} y\right)\right),
\end{aligned}
$$

where $A_{m n}, d_{l m}^{1}, f_{l n}^{1}, B_{m n}, d_{l m}^{2}, f_{l n}^{2}, C_{m n}, d_{l m}^{3}$, and $f_{l n}^{3}$ are the expansion coefficients, $\lambda_{m}=m \pi / a, \lambda_{n}=n \pi / b, a$ and $b$ are the length and width, respectively, and

$$
\begin{aligned}
& \xi_{1 a}(x)=\frac{a}{2 \pi} \sin \frac{\pi x}{2 a}+\frac{a}{2 \pi} \sin \frac{3 \pi x}{2 a}, \\
& \xi_{2 a}(x)=-\frac{a}{2 \pi} \cos \frac{\pi x}{2 a}+\frac{a}{2 \pi} \cos \frac{3 \pi x}{2 a} \\
& \xi_{1 b}(y)=\frac{b}{2 \pi} \sin \frac{\pi y}{2 b}+\frac{b}{2 \pi} \sin \frac{3 \pi y}{2 b} \\
& \xi_{2 b}(y)=-\frac{b}{2 \pi} \cos \frac{\pi y}{2 b}+\frac{b}{2 \pi} \cos \frac{3 \pi y}{2 b} .
\end{aligned}
$$

Theoretically, there is an infinite number of these supplementary functions. However, one needs to ensure that the selected functions will not nullify any of the boundary conditions. It is easy to verify that $\xi 1 a(0)=\xi 1 a(a)=\xi 1 a^{\prime}(a)=0$, $\xi 1 a^{\prime}(0)=1, \xi 2 a(0)=\xi 2 a(a)=\xi 2 a^{\prime}(0)=0, \xi 2 a^{\prime}(a)=1$, similar conditions exist for the supplementary function in $y$ direction. Though these conditions are not necessary, they can simplify the subsequent mathematical expressions and the corresponding solution procedures.

One will notice from (4) that beside the standard double Fourier series, four single Fourier series are also included. The potential discontinuity associated with the $x$-derivative and $y$-derivative of the original function along the four edges can be transferred onto these auxiliary series functions. Then, the Fourier series would be smooth enough in the whole solving domain. Therefore, not only is this Fourier series representation of solution applicable to any boundary conditions but also the convergence of the series expansion can be improved.

Substituting (4) into the boundary conditions, for example, $k_{x 0} w=-Q_{x}$, at $x=0$; one can have

$$
\begin{aligned}
& k_{x 0}\left(\sum_{m=0}^{\infty} \sum_{n=0}^{\infty} A_{m n} \cos \lambda_{n} y+\xi_{1 b}(y) \sum_{m=0}^{\infty} d_{1 m}^{1}\right. \\
& \left.\quad+\xi_{2 b}(y) \sum_{m=0}^{\infty} d_{2 m}^{1}\right)
\end{aligned}
$$

$$
\begin{aligned}
=-k G h\left(\sum_{n=0}^{\infty} f_{1 n}^{1} \cos \lambda_{n} y+\sum_{m=0}^{\infty} \sum_{n=0}^{\infty} B_{m n} \cos \lambda_{n} y\right. \\
\left.+\xi_{1 b}(y) \sum_{m=0}^{\infty} d_{1 m}^{2}+\xi_{2 b}(y) \sum_{m=0}^{\infty} d_{2 m}^{2}\right) .
\end{aligned}
$$

In order to derive the constraint equations for the unknown coefficients, all the sine terms and the auxiliary series functions will be expanded into Fourier cosine series. The related formulas are provided in Appendix A. Then, by equating the coefficients for the like terms on both sides, one can obtain the following equations:

$$
\begin{gathered}
k_{x 0}\left(\sum_{m=0}^{\infty} A_{m n}+\beta_{1 n} \sum_{m=0}^{\infty} d_{1 m}^{1}+\beta_{2 n} \sum_{m=0}^{\infty} d_{2 m}^{1}\right) \\
=-k G h\left(f_{1 n}^{1}+\sum_{m=0}^{\infty} B_{m n}+\beta_{1 n} \sum_{m=0}^{\infty} d_{1 m}^{2}\right. \\
\left.+\beta_{2 n} \sum_{m=0}^{\infty} d_{2 m}^{2}\right) .
\end{gathered}
$$

Similarly, the substitution of (4) into the remaining boundary conditions will lead to eleven equations that can be obtained from (3):

$$
\begin{aligned}
& K_{x 0}\left(\sum_{m=0}^{\infty} B_{m n}+\beta_{1 n} \sum_{m=0}^{\infty} d_{1 m}^{2}+\beta_{2 n} \sum_{m=0}^{\infty} d_{2 m}^{2}\right) \\
& =-D\left(f_{1 n}^{2}\right. \\
& +\mu\left(\sum_{m=0}^{\infty} \sum_{q=0}^{\infty}\left(-\lambda_{q}\right) C_{m q} \tau_{q}\right. \\
& \left.\left.+\eta_{1 n} \sum_{m=0}^{\infty} d_{1 m}^{3}+\eta_{2 n} \sum_{m=0}^{\infty} d_{2 m}^{3}\right)\right) \\
& K_{y x 0}\left(\sum_{m=0}^{\infty} C_{m n}+\beta_{1 n} \sum_{m=0}^{\infty} d_{1 m}^{3}+\beta_{2 n} \sum_{m=0}^{\infty} d_{2 m}^{3}\right) \\
& =-\frac{1-\mu}{2} D\left(f_{1 n}^{3}+\sum_{m=0}^{\infty} \sum_{q=0}^{\infty}\left(-\lambda_{q}\right) B_{m q} \tau_{q}\right. \\
& \left.+\eta_{1 n} \sum_{m=0}^{\infty} d_{1 m}^{2}+\eta_{2 n} \sum_{m=0}^{\infty} d_{2 m}^{2}\right) \\
& k_{x a}\left(\sum_{m=0}^{\infty}(-1)^{m} A_{m n}+\sum_{l=1}^{2}\left(\beta_{l n} \sum_{m=0}^{\infty}(-1)^{m} d_{l m}^{1}\right)\right) \\
& =k G h\left(f_{2 n}^{1}+\sum_{m=0}^{\infty}(-1)^{m} B_{m n}+\sum_{l=1}^{2}\left(\beta_{l n} \sum_{m=0}^{\infty}(-1)^{m} d_{l m}^{2}\right)\right) \text {, }
\end{aligned}
$$




$$
\begin{array}{cc}
K_{x a}\left(\sum_{m=0}^{\infty}(-1)^{m} B_{m n}+\sum_{l=1}^{2}\left(\beta_{l n} \sum_{m=0}^{\infty}(-1)^{m} d_{l m}^{2}\right)\right) & K_{y b}\left(\sum_{n=0}^{\infty}(-1)^{n} C_{m n}+\sum_{l=1}^{2}\left(\alpha_{l m} \sum_{n=0}^{\infty}(-1)^{n} f_{l n}^{3}\right)\right) \\
=-D\left(f_{2 n}^{2}+\mu\left(\sum_{m=0}^{\infty} \sum_{q=0}^{\infty}\left(-\lambda_{q}\right)(-1)^{m} C_{m q} \tau_{q}\right.\right. & =D\left(d_{2 m}^{3}+\mu\left(\sum_{n=0}^{\infty} \sum_{p=0}^{\infty}\left(-\lambda_{p}\right)(-1)^{n} B_{m p} \varepsilon_{p}\right.\right. \\
\left.\left.+\sum_{l=1}^{2}\left(\eta_{l n} \sum_{m=0}^{\infty}(-1)^{m} d_{l m}^{3}\right)\right)\right), & \left.\left.+\sum_{l=1}^{2}\left(\gamma_{l m} \sum_{n=0}^{\infty}(-1)^{n} f_{l n}^{2}\right)\right)\right), \\
K_{y x a}\left(\sum_{m=0}^{\infty}(-1)^{m} C_{m n}+\sum_{l=1}^{2}\left(\beta_{l n} \sum_{m=0}^{\infty}(-1)^{m} d_{l m}^{3}\right)\right) & K_{x y b}\left(\sum_{n=0}^{\infty}(-1)^{n} B_{m n}+\sum_{l=1}^{2}\left(\alpha_{l m} \sum_{n=0}^{\infty}(-1)^{n} f_{l n}^{2}\right)\right) \\
=\frac{1-\mu}{2} D\left(f_{2 n}^{3}+\sum_{m=0}^{\infty} \sum_{q=0}^{\infty}\left(-\lambda_{q}\right)(-1)^{m} B_{m q} \tau_{q}\right. & =\frac{1-\mu}{2} D\left(d_{2 m}^{2}+\sum_{n=0}^{\infty} \sum_{p=0}^{\infty}\left(-\lambda_{p}\right)(-1)^{n} C_{p n} \varepsilon_{p}\right. \\
\left.+\sum_{l=1}^{2}\left(\eta_{l n} \sum_{m=0}^{\infty}(-1)^{m} d_{l m}^{2}\right)\right), & \left.+\sum_{l=1}^{2}\left(\gamma_{l m} \sum_{n=0}^{\infty}(-1)^{n} f_{l n}^{3}\right)\right) .
\end{array}
$$$$
k_{y 0}\left(\sum_{n=0}^{\infty} A_{m n}+\alpha_{1 m} \sum_{n=0}^{\infty} f_{1 n}^{1}+\alpha_{2 m} \sum_{n=0}^{\infty} f_{2 n}^{1}\right)
$$$$
=-k G h\left(d_{1 m}^{1}+\sum_{n=0}^{\infty} C_{m n}+\alpha_{1 m} \sum_{n=0}^{\infty} f_{1 n}^{3}+\alpha_{2 m} \sum_{n=0}^{\infty} f_{2 n}^{3}\right) \text {, }
$$$$
K_{y 0}\left(\sum_{n=0}^{\infty} C_{m n}+\alpha_{1 m} \sum_{n=0}^{\infty} f_{1 n}^{3}+\alpha_{2 m} \sum_{n=0}^{\infty} f_{2 n}^{3}\right)
$$$$
=-D\left(d_{1 m}^{3}\right.
$$$$
+\mu\left(\sum_{n=0}^{\infty} \sum_{p=0}^{\infty}\left(-\lambda_{p}\right) B_{m p} \varepsilon_{p}\right.
$$$$
\left.\left.+\gamma_{1 m} \sum_{n=0}^{\infty} f_{1 n}^{2}+\gamma_{2 m} \sum_{n=0}^{\infty} f_{2 n}^{2}\right)\right)
$$$$
K_{x y 0}\left(\sum_{n=0}^{\infty} B_{m n}+\alpha_{1 m} \sum_{n=0}^{\infty} f_{1 n}^{2}+\alpha_{2 m} \sum_{n=0}^{\infty} f_{2 n}^{2}\right)
$$$$
=-\frac{1-\mu}{2} D\left(d_{1 m}^{2}+\sum_{n=0}^{\infty} \sum_{p=0}^{\infty}\left(-\lambda_{p}\right) C_{p n} \varepsilon_{p}\right.
$$$$
\left.+\gamma_{1 m} \sum_{n=0}^{\infty} f_{1 n}^{3}+\gamma_{2 m} \sum_{n=0}^{\infty} f_{2 n}^{3}\right)
$$$$
k_{y b}\left(\sum_{n=0}^{\infty}(-1)^{n} A_{m n}+\sum_{l=1}^{2}\left(\alpha_{l m} \sum_{n=0}^{\infty}(-1)^{n} f_{l n}^{1}\right)\right)
$$$$
=k G h\left(d_{2 m}^{1}+\sum_{n=0}^{\infty}(-1)^{n} C_{m n}+\sum_{l=1}^{2}\left(\alpha_{l m} \sum_{n=0}^{\infty}(-1)^{n} f_{l n}^{3}\right)\right) \text {, }
$$

When all the series expansions are truncated to $m=M$ and $n=N$ in numerical calculations, the twelve equations can be rewritten in a matrix form as

$$
\mathbf{H B}=\mathbf{Q A},
$$

where

$$
\begin{aligned}
& \mathbf{H}=\left[\begin{array}{cccc}
\mathbf{H}_{1,1} & \mathbf{H}_{1,2} & \cdots & \mathbf{H}_{1,12} \\
\mathbf{H}_{2,1} & \mathbf{H}_{2,2} & \cdots & \mathbf{H}_{2,12} \\
\vdots & \vdots & \ddots & \vdots \\
\mathbf{H}_{12,1} & \mathbf{H}_{12,2} & \cdots & \mathbf{H}_{12,12}
\end{array}\right], \\
& \mathbf{Q}=\left[\begin{array}{ccc}
\mathbf{Q}_{1,1} & \mathbf{Q}_{1,2} & \mathbf{Q}_{1,3} \\
\mathbf{Q}_{2,1} & \mathbf{Q}_{2,2} & \mathbf{Q}_{2,3} \\
\vdots & \vdots & \vdots \\
\mathbf{Q}_{12,1} & \mathbf{Q}_{12,2} & \mathbf{Q}_{12,3}
\end{array}\right], \\
& \mathbf{A}=\left\{A_{00}, A_{01}, \ldots, A_{M N}, B_{00}, B_{01}, \ldots, B_{M N},\right. \\
& \left.C_{00}, C_{01}, \ldots, C_{M N}\right\}^{T} \text {, } \\
& \mathbf{B}=\left\{d_{10}^{1}, d_{11}^{1}, \ldots, d_{2 M}^{1}, f_{10}^{1}, f_{11}^{1}, \ldots, f_{2 N}^{1}, d_{10}^{2}, d_{11}^{2}, \ldots, d_{2 M}^{2},\right. \\
& f_{10}^{2}, f_{11}^{2}, \ldots, f_{2 N}^{2}, d_{10}^{3} \text {, } \\
& \left.d_{11}^{3}, \ldots, d_{2 M}^{3}, f_{10}^{3}, f_{11}^{3}, \ldots, f_{2 N}^{3}\right\}^{T} \text {. }
\end{aligned}
$$

The elements of the matrices $\mathbf{H}$ and $\mathbf{Q}$ are defined in Appendix B.

By substituting (4) into the governing differential equation (1), as mentioned earlier, all the sine terms and the auxiliary series functions are expanded into Fourier cosine series. 
Then, by equating the coefficients for the like terms on both sides, one can obtain the following equations:

$$
\begin{aligned}
& A_{m n}\left(-\lambda_{m}^{2}\right) \\
& +\sum_{l=1}^{2}\left(d_{l m}^{1}\left(-\lambda_{m}^{2}\right) \beta_{l n}+f_{l n}^{1} \phi_{l m}\right)+A_{m n}\left(-\lambda_{n}^{2}\right) \\
& +\sum_{l=1}^{2}\left(d_{l m}^{1} \varphi_{l n}+\left(-\lambda_{n}^{2}\right) f_{l n}^{1} \alpha_{l m}\right)+\sum_{p=0}^{\infty} B_{m n}\left(-\lambda_{m}\right) \varepsilon_{m p} \\
& +\sum_{l=1}^{2}\left(\sum_{p=0}^{\infty} d_{l m}^{2}\left(-\lambda_{m}\right) \varepsilon_{m p} \beta_{l n}+f_{l n}^{2} \gamma_{l m}\right) \\
& +\sum_{q=0}^{\infty} C_{m n}\left(-\lambda_{n}\right) \tau_{n q}+\sum_{l=1}^{2}\left(d_{l m}^{3} \eta_{l n}+\sum_{q=0}^{\infty} f_{l n}^{3}\left(-\lambda_{n}\right) \tau_{n q} \alpha_{l m}\right) \\
& +\frac{\rho \omega^{2}}{k G}\left(A_{m n}+\beta_{1 n} d_{1 m}^{1}+f_{1 n}^{1} \alpha_{1 m}+\beta_{2 n} d_{2 m}^{1}+f_{2 n}^{1} \alpha_{2 m}\right)=0, \\
& D\left(B_{m n}\left(-\lambda_{m}^{2}\right)+\sum_{l=1}^{2}\left(\beta_{l n} d_{l m}^{2}\left(-\lambda_{m}^{2}\right)+\phi_{l m} f_{l n}^{2}\right)\right. \\
& +\frac{1-\mu}{2}\left(B_{m n}\left(-\lambda_{n}^{2}\right)\right. \\
& \left.+\sum_{l=1}^{2}\left(\varphi_{l n} d_{l m}^{2}+\alpha_{l m} f_{l n}^{2}\left(-\lambda_{n}^{2}\right)\right)\right) \\
& +\frac{1+\mu}{2}\left(\sum_{p=0}^{\infty} \sum_{q=0}^{\infty} C_{m n} \lambda_{m} \lambda_{n} \varepsilon_{m p} \tau_{n q}\right. \\
& +\sum_{l=1}^{2}\left(\sum_{p=0}^{\infty} d_{l m}^{3} \eta_{l n}\left(-\lambda_{m}\right) \varepsilon_{m p}\right. \\
& \left.\left.\left.+\sum_{q=0}^{\infty} f_{l n}^{3} \gamma_{l m}\left(-\lambda_{n}\right) \tau_{n q}\right)\right)\right)
\end{aligned}
$$$$
-k G h\left(\sum_{p=0}^{\infty} A_{m n}\left(-\lambda_{m}\right) \varepsilon_{m p}\right.
$$$$
+\sum_{l=1}^{2}\left(\sum_{p=0}^{\infty} \beta_{l n} d_{l m}^{1}\left(-\lambda_{m}\right) \varepsilon_{m p}+\gamma_{l n} f_{l n}^{1}\right)
$$$$
\left.+B_{m n}+\sum_{l=1}^{2}\left(\beta_{l n} d_{l m}^{2}+\alpha_{l m} f_{l n}^{2}\right)\right)
$$$$
+\rho h \omega^{2}\left(B_{m n}+\sum_{l=1}^{2}\left(\beta_{l n} d_{l m}^{2}+\alpha_{l m} f_{l n}^{2}\right)\right)=0,
$$

$$
\begin{gathered}
D\left(C_{m n}\left(-\lambda_{n}^{2}\right)+\sum_{l=1}^{2}\left(\varphi_{l n} d_{l m}^{3}+\alpha_{l m} f_{l n}^{3}\left(-\lambda_{n}^{2}\right)\right)\right. \\
+\frac{1-\mu}{2}\left(C_{m n}\left(-\lambda_{m}^{2}\right)\right. \\
\left.+\sum_{l=1}^{2}\left(\beta_{l n} d_{l m}^{3}\left(-\lambda_{m}^{2}\right)+\phi_{l m} f_{l n}^{3}\right)\right) \\
+\frac{1+\mu}{2}\left(\sum_{p=0}^{\infty} \sum_{q=0}^{\infty} B_{m n} \lambda_{m} \lambda_{n} \varepsilon_{m p} \tau_{n q}\right. \\
+\sum_{l=1}^{2}\left(\sum_{p=0}^{\infty} d_{l m}^{2} \eta_{l n}\left(-\lambda_{m}\right) \varepsilon_{m p}\right. \\
\left.+k G h\left(\sum_{q=0}^{\infty} A_{m n}\left(-\lambda_{n}\right) \tau_{n q}^{2} f_{l n}^{2} \gamma_{l m}\left(-\lambda_{n}\right) \tau_{n q}\right)\right) \\
+\sum_{l=1}^{2}\left(d_{l m}^{1} \eta_{l n}+\sum_{q=0}^{\infty} f_{l n}^{1} \alpha_{l m}\left(-\lambda_{n}\right) \tau_{n q}\right) \\
\left.+C_{m n}+\sum_{l=1}^{2}\left(d_{l m}^{3} \beta_{l n}+f_{l n}^{3} \alpha_{l m}\right)\right) \\
+\rho h \omega^{2}\left(C_{m n}+\sum_{l=1}^{2}\left(d_{l m}^{3} \beta_{l n}+f_{l n}^{3} \alpha_{l m}\right)\right)=0 .
\end{gathered}
$$

Writing in matrix form, we have

$$
\mathbf{C A}+\mathbf{D B}+\frac{\rho h \omega^{2}}{k G}(\mathbf{E A}+\mathbf{F B})=\mathbf{0}
$$

Substituting (9), the final system equations can be obtained as

$$
\left(\mathbf{K}+\frac{\rho h \omega^{2}}{k G} \mathbf{M}\right) \mathbf{A}=\mathbf{0},
$$

where

$$
\begin{gathered}
\mathbf{C}=\left[\begin{array}{lll}
\mathbf{C}_{1,1} & \mathbf{C}_{1,2} & \mathbf{C}_{1,3} \\
\mathbf{C}_{2,1} & \mathbf{C}_{2,2} & \mathbf{C}_{2,3} \\
\mathbf{C}_{3,1} & \mathbf{C}_{3,2} & \mathbf{C}_{3,3}
\end{array}\right], \\
\mathbf{D}=\left[\begin{array}{llll}
\mathbf{D}_{1,1} & \mathbf{D}_{1,2} & \cdots & \mathbf{D}_{1,12} \\
\mathbf{D}_{2,1} & \mathbf{D}_{2,2} & \cdots & \mathbf{D}_{2,12} \\
\mathbf{D}_{3,1} & \mathbf{D}_{3,2} & \cdots & \mathbf{D}_{3,12}
\end{array}\right], \\
\mathbf{E}=\left[\begin{array}{lll}
\mathbf{E}_{1,1} & \mathbf{E}_{1,2} & \mathbf{E}_{1,3} \\
\mathbf{E}_{2,1} & \mathbf{E}_{2,2} & \mathbf{E}_{2,3} \\
\mathbf{E}_{3,1} & \mathbf{E}_{3,2} & \mathbf{E}_{3,3}
\end{array}\right],
\end{gathered}
$$


TABLE 1: The first seven frequency parameters $\Omega=\left(\omega b^{2} / \pi^{2}\right)(\rho h / D)^{1 / 2}$ for F-F-F-F Mindlin rectangular plates.

\begin{tabular}{lccccccc}
\hline$M=N$ & 1 & 2 & 3 & 4 & 5 & 6 & 7 \\
\hline 5 & 1.2978 & 1.9202 & 2.3644 & 3.2556 & 3.2556 & 5.6148 & 5.6148 \\
7 & 1.2924 & 1.9196 & 2.3637 & 3.2438 & 3.2438 & 5.6105 & 5.6105 \\
9 & 1.2904 & 1.9195 & 2.3635 & 3.2388 & 3.2388 & 5.6092 \\
10 & 1.2903 & 1.9194 & 2.3634 & 3.2371 & 3.2371 & 5.6089 & 5.6092 \\
11 & 1.2895 & 1.9194 & 2.3634 & 3.2366 & 3.2366 & 5.6087 & 5.6087 \\
12 & 1.2895 & 1.9194 & 2.3633 & 3.2358 & 3.2358 & 5.6085 \\
13 & 1.2892 & 1.9194 & 2.3633 & 3.2355 & 3.2355 & 5.6085 & 5.6085 \\
14 & 1.2892 & 1.9194 & 2.3633 & 3.2355 & 3.2355 & 5.6084 & 5.6084 \\
\hline
\end{tabular}

TABLE 2: The first seven frequency parameters $\Omega=\left(\omega a^{2}\right)(\rho h / D)^{1 / 2}$ for S-F-S-F Mindlin rectangular plates.

\begin{tabular}{|c|c|c|c|c|c|c|c|c|c|}
\hline$a / b$ & $h / a$ & Method & 1 & 2 & 3 & 4 & 5 & 6 & 7 \\
\hline \multirow{4}{*}{0.4} & \multirow{2}{*}{0.1} & Present & 9.5829 & 10.7868 & 14.5793 & 20.7593 & 29.3643 & 36.7449 & 37.8085 \\
\hline & & [9] & 9.5814 & 10.7809 & 14.5672 & 20.7245 & 29.3338 & 36.736 & 37.7879 \\
\hline & \multirow{2}{*}{0.2} & Present & 9.1316 & 10.1983 & 13.5314 & 18.7785 & 25.8073 & 31.7024 & 32.4788 \\
\hline & & [9] & 9.1313 & 10.1968 & 13.5287 & 18.7728 & 25.8016 & 31.7000 & 32.4730 \\
\hline \multirow{4}{*}{1} & \multirow{2}{*}{0.1} & Present & 9.4461 & 15.4109 & 33.9251 & 36.4266 & 42.8986 & 62.3508 & 66.3889 \\
\hline & & [9] & 9.4458 & 15.4054 & 33.9160 & 36.4246 & 42.8870 & 62.3304 & 66.3720 \\
\hline & \multirow{2}{*}{0.2} & Present & 8.9997 & 14.1349 & 29.2566 & 31.434 & 36.1663 & 49.8976 & 52.8026 \\
\hline & & [9] & 8.9997 & 14.1341 & 29.2558 & 31.4338 & 36.1646 & 49.8953 & 52.8012 \\
\hline \multirow{4}{*}{2.5} & \multirow{2}{*}{0.1} & Present & 9.3065 & 29.6407 & 35.8486 & 64.0871 & 75.9262 & 105.417 & 125.3433 \\
\hline & & [9] & 9.3065 & 29.6389 & 35.8486 & 64.0850 & 75.9234 & 105.4121 & 125.3334 \\
\hline & \multirow{2}{*}{0.2} & Present & 8.8835 & 24.2058 & 30.9976 & 49.7612 & 59.4412 & 77.0664 & 90.361 \\
\hline & & [9] & 8.8835 & 24.2054 & 30.9976 & 49.7609 & 59.4404 & 77.0654 & 90.3658 \\
\hline
\end{tabular}

$$
\mathbf{F}=\left[\begin{array}{llll}
\mathbf{F}_{1,1} & \mathbf{F}_{1,2} & \cdots & \mathbf{F}_{1,12} \\
\mathbf{F}_{2,1} & \mathbf{F}_{2,2} & \cdots & \mathbf{F}_{2,12} \\
\mathbf{F}_{3,1} & \mathbf{F}_{3,2} & \cdots & \mathbf{F}_{3,12}
\end{array}\right],
$$

where $\mathbf{K}=\mathbf{C}+\mathbf{D H}^{-1} \mathbf{Q}$ and $\mathbf{M}=\mathbf{E}+\mathbf{F H}^{-1} \mathbf{Q}$. The element of the matrices $\mathbf{C}, \mathbf{D}, \mathbf{E}$, and $\mathbf{F}$ are defined in Appendix B. The natural frequencies and eigenvectors can be obtained through solving (13). Then, the physical mode shapes also can be got using (4) and (9).

\section{Result and Discussion}

Several examples involving various boundary conditions will be discussed in this section. To avoid any comparison of the roundoff results, which might be unrealistic, the nondimensional frequency is used. For the analysis, Poisson's ratio $\mu=$ 0.3 and shear correction factor $k=5 / 6$ are used.

First, convergence studies are carried out. Table 1 gives the frequencies calculated by using different number of terms in the series expansion for F-F-F-F Mindlin rectangular plates (for $a / b=1$ and $h / a=0.1$ ). It shows that the results are very accurate when $M$ and $N$ are small numbers. When $M$ and $N$ are larger than 10 , results are almost invariant. So this method has a very good convergence characteristic.

In order to evaluate the accuracy of the present method, the comparisons with those results reported in the literature are carried out. First of all, considering a plate with two opposite simple edges and the other two edges free (S-F-S-F).
A simple edge can be obtained by setting the translational and torsional spring constants to be infinite (here the infinite is represented by a very large number, $D \times 10^{7}$, and $D$ is the flexural rigidity) and the rotational spring constants to be zero. A free edge can be obtained when all the spring constants are zero. In Table 2 , the first seven nondimensional frequency parameters, $\Omega=\left(\omega a^{2}\right)(\rho h / D)^{1 / 2}$, are given with different aspect ratios and thickness ratios. At the same time, a comparison of the exact solution in [9] is also presented. The results show that the calculated frequencies are excellent. As mentioned earlier, the series expansion will have to be truncated in numerical calculations. In this example and all the subsequent calculations, the setting $M=N=12$ is used. Specially, in order to compare with the results in [9], shear correction factor $k=0.86667$ is used.

Three more classical cases (C-F-F-F, C-F-S-F, and C$\mathrm{S}-\mathrm{S}-\mathrm{F})$ were considered, and the corresponding frequency parameters are listed in Tables 3, 4, and 5. A clamped edge can be viewed as all the spring constants set to be infinite. The frequency parameters solved by Ritz method and DSC method also are given as a comparison. A good agreement is also observed among these solutions.

This method not only can solve the Mindlin rectangular plates with classical boundary conditions but also can solve plates with the elastical supports. Xiang et al. [3] and Gorman [11] and Zhou [4] also researched Mindlin plates with elastically restrained edges, but they both used two kinds of springs (rotational and translational springs) to realize the elastical 
TABLE 3: The first seven frequency parameters $\Omega=\left(\omega b^{2} / \pi^{2}\right)(\rho h / D)^{1 / 2}$ for C-F-F-F Mindlin rectangular plates.

\begin{tabular}{|c|c|c|c|c|c|c|c|c|c|}
\hline$a / b$ & $h / b$ & Method & 1 & 2 & 3 & 4 & 5 & 6 & 7 \\
\hline \multirow{4}{*}{0.4} & \multirow{2}{*}{0.1} & Present & 2.1006 & 2.7297 & 4.3933 & 7.2648 & 10.4212 & 11.1567 & 11.4449 \\
\hline & & [1] & 2.1006 & 2.7294 & 4.3921 & 7.2622 & 10.4206 & 11.1554 & 11.4433 \\
\hline & \multirow{2}{*}{0.2} & Present & 1.8579 & 2.2911 & 3.4911 & 5.5123 & 6.9021 & 7.3873 & 8.077 \\
\hline & & [1] & 1.8579 & 2.2910 & 3.4909 & 5.5119 & 6.9020 & 7.3871 & 8.0767 \\
\hline \multirow{4}{*}{1} & \multirow{2}{*}{0.1} & Present & 0.3476 & 0.8171 & 2.0357 & 2.584 & 2.8633 & 4.8186 & 5.4836 \\
\hline & & [1] & 0.3476 & 0.8168 & 2.0356 & 2.5836 & 2.8620 & 4.8162 & 5.4834 \\
\hline & \multirow{2}{*}{0.2} & Present & 0.3384 & 0.7446 & 1.7806 & 2.2766 & 2.4207 & 3.8853 & 4.3156 \\
\hline & & [1] & 0.3384 & 0.7445 & 1.7806 & 2.2765 & 2.4205 & 3.8851 & 4.3168 \\
\hline \multirow{4}{*}{2.5} & \multirow{2}{*}{0.1} & Present & 0.0554 & 0.2798 & 0.3432 & 0.8865 & 0.9517 & 1.6251 & 1.8326 \\
\hline & & {$[1]$} & 0.0554 & 0.2795 & 0.3431 & 0.8854 & 0.9516 & 1.6228 & 1.8320 \\
\hline & \multirow{2}{*}{0.2} & Present & 0.0550 & 0.2626 & 0.3337 & 0.8196 & 0.8958 & 1.4639 & 1.6566 \\
\hline & & [1] & 0.0550 & 0.2625 & 0.3337 & 0.8192 & 0.8958 & 1.4632 & 1.6564 \\
\hline
\end{tabular}

TABLE 4: The first seven frequency parameters $\Omega=\left(\omega b^{2} / \pi^{2}\right)(\rho h / D)^{1 / 2}$ for C-F-S-F Mindlin rectangular plates.

\begin{tabular}{|c|c|c|c|c|c|c|c|c|c|}
\hline$a / b$ & $h / b$ & Method & 1 & 2 & 3 & 4 & 5 & 6 & 7 \\
\hline \multirow{4}{*}{0.4} & \multirow{2}{*}{0.1} & Present & 7.8209 & 8.2262 & 9.6485 & 12.1337 & 15.6899 & 20.1409 & 20.358 \\
\hline & & {$[8]$} & 7.8285 & 8.2339 & 9.6566 & 12.1373 & 15.6917 & 20.1394 & 20.3607 \\
\hline & \multirow{2}{*}{0.2} & Present & 5.5099 & 5.7895 & 6.7405 & 8.3478 & 10.4967 & 12.6125 & 12.8205 \\
\hline & & [8] & 5.5128 & 5.7926 & 6.7436 & 8.3495 & 10.4978 & 12.6141 & 12.8219 \\
\hline \multirow{4}{*}{1} & \multirow{2}{*}{0.1} & Present & 1.4735 & 1.9497 & 3.6462 & 4.502 & 5.0407 & 6.7828 & 6.8161 \\
\hline & & [7] & 1.4738 & 1.9536 & 3.6499 & 4.5031 & 5.0483 & 6.7909 & - \\
\hline & \multirow{2}{*}{0.2} & Present & 1.3254 & 1.7019 & 3.0527 & 3.6262 & 4.0033 & 5.2067 & 5.3485 \\
\hline & & [7] & 1.3255 & 1.7030 & 3.0534 & 3.6265 & 4.0054 & 5.2084 & - \\
\hline \multirow{4}{*}{2.5} & \multirow{2}{*}{0.1} & Present & 0.2413 & 0.5789 & 0.776 & 1.2825 & 1.5996 & 2.1811 & 2.4288 \\
\hline & & [7] & 0.2413 & 0.5817 & 0.7761 & 1.2859 & 1.5995 & 2.1852 & - \\
\hline & \multirow{2}{*}{0.2} & Present & 0.2362 & 0.5402 & 0.7365 & 1.1704 & 1.4595 & 1.9285 & 2.1794 \\
\hline & & [7] & 0.2362 & 0.5409 & 0.7366 & 1.1714 & 1.4594 & 1.9300 & - \\
\hline
\end{tabular}

TABLE 5: The first seven frequency parameters $\Omega=\left(\omega b^{2} / \pi^{2}\right)(\rho h / D)^{1 / 2}$ for C-S-S-F Mindlin rectangular plates.

\begin{tabular}{|c|c|c|c|c|c|c|c|c|c|}
\hline$a / b$ & $h / b$ & Method & 1 & 2 & 3 & 4 & 5 & 6 & 7 \\
\hline \multirow{4}{*}{0.4} & \multirow{2}{*}{0.1} & Present & 7.9414 & 8.9713 & 11.137 & 14.4651 & 18.7651 & 20.4622 & 21.36 \\
\hline & & {$[8]$} & 7.9491 & 8.9792 & 11.1430 & 14.4679 & 18.7554 & 20.4646 & 21.3629 \\
\hline & \multirow{2}{*}{0.2} & Present & 5.5943 & 6.3055 & 7.752 & 9.8284 & 12.295 & 12.6792 & 13.2365 \\
\hline & & [8] & 5.5972 & 6.3085 & 7.7544 & 9.8298 & 12.2958 & 12.6807 & 13.2381 \\
\hline \multirow{4}{*}{1} & \multirow{2}{*}{0.1} & Present & 1.6196 & 2.9171 & 4.6617 & 5.7683 & 5.9723 & 8.5764 & 8.8548 \\
\hline & & [8] & 1.6281 & 2.9271 & 4.6650 & 5.7741 & 5.9769 & 8.5795 & 9.8555 \\
\hline & \multirow{2}{*}{0.2} & Present & 1.4451 & 2.4997 & 3.7407 & 4.6356 & 4.677 & 6.4325 & 6.4907 \\
\hline & & [8] & 1.4476 & 2.5027 & 3.7419 & 4.6375 & 4.6787 & 6.4338 & 6.4910 \\
\hline \multirow{4}{*}{2.5} & \multirow{2}{*}{0.1} & Present & 0.3658 & 0.9415 & 1.746 & 1.7792 & 2.3445 & 2.8701 & 3.217 \\
\hline & & {$[8]$} & 0.3832 & 0.9487 & 1.7596 & 1.7828 & 2.3543 & 2.8721 & 3.2233 \\
\hline & \multirow{2}{*}{0.2} & Present & 0.3526 & 0.8834 & 1.6006 & 1.6092 & 2.0839 & 2.4914 & 2.7667 \\
\hline & & {$[8]$} & 0.3574 & 0.8854 & 1.6043 & 1.6102 & 2.0867 & 2.4919 & 2.7685 \\
\hline
\end{tabular}

supports along every edge. As mentioned earlier, three kinds of springs along every edge are needed to truly realize the general elastic supports, including all classical homogeneous boundary conditions. Table 6 gives the first seven frequency parameters for Mindlin rectangular plates (for $a / b=1$ and $h / a=0.2$ ) with two opposite free edges, the other two edges only elastically restrained against translation, and Table 7 gives the first seven frequency parameters for the plates with two opposite free edges, the other two edges symmetrically elastically restrained. Table 8 lists the frequency parameters when the translational, rotational, and torsional spring constants are all varying at $x=a$. For simplicity, all the restraining springs are assumed to have the same stiffness in the three examples. Table 8 shows that the results are invariant when $K$ is larger than $10^{7}$, so it is appropriate to set infinite to be $D \times 10^{7}$ in this paper. 
TABLE 6: The first seven frequency parameters $\Omega=\left(\omega b^{2} / \pi^{2}\right)(\rho h / D)^{1 / 2}$ for Mindlin rectangular plates with two opposite free edges, the other two edges only elastically restrained against translation $k_{x 0}=k_{x a}=K \times D$.

\begin{tabular}{lcccccccc}
\hline$K$ & Method & 1 & 2 & 3 & 4 & 5 & 6 & 7 \\
\hline \multirow{2}{*}{5} & Present & 0.3051 & 0.3071 & 0.5397 & 1.2922 & 1.8248 & 2.2009 & 2.8356 \\
& {$[4]$} & 0.3052 & 0.3075 & 0.5396 & 1.3117 & 1.8447 & 2.2089 & 2.8913 \\
\hline \multirow{2}{*}{50} & Present & 0.6926 & 0.8072 & 1.5785 & 1.9220 & 2.1374 & 2.7775 \\
& {$[4]$} & 0.6941 & 0.8225 & 1.5797 & 1.9268 & 2.1634 & 2.7919 & 3.3443 \\
\multirow{2}{*}{500} & Present & 0.8782 & 1.2203 & 2.6177 & 2.8662 & 3.1461 & 4.2179 \\
& {$[4]$} & 0.8831 & 1.3081 & 2.7583 & 2.8767 & 3.2089 & 4.3584 & 5.0805 \\
\multirow{2}{*}{5000} & Present & 0.905 & 1.306 & 2.7851 & 3.1319 & 3.4845 & 4.7069 & 5.1891 \\
& {$[4]$} & 0.9105 & 1.4254 & 2.9541 & 3.1485 & 3.6157 & 4.9856 & 5.3230 \\
\hline
\end{tabular}

TABLE 7: The first seven frequency parameters $\Omega=\left(\omega b^{2} / \pi^{2}\right)(\rho h / D)^{1 / 2}$ for Mindlin rectangular plates with two opposite free edges, the other two edges symmetrically elastically restrained $k_{x 0}=k_{x a}=K_{1} \times D$ and $K_{x 0}=K_{x a}=K_{2} \times D$.

\begin{tabular}{lccccccccc}
\hline$K_{1}$ & $K_{2}$ & Method & 1 & 2 & 3 & 4 & 5 & 6 & 7 \\
\hline \multirow{2}{*}{5} & \multirow{2}{*}{10} & Present & 0.3105 & 0.3148 & 0.9155 & 1.4411 & 2.006 & 2.8874 & 2.9385 \\
& & {$[4]$} & 0.3104 & 0.3148 & 0.9208 & 1.4505 & 2.0122 & 2.8989 & 2.9659 \\
\hline \multirow{2}{*}{50} & \multirow{2}{*}{50} & Present & 0.8936 & 0.901 & 1.6281 & 1.9545 & 2.1865 & 3.2206 & 3.412 \\
& & {$[4]$} & 0.8944 & 0.9032 & 1.6305 & 1.9599 & 2.1912 & 3.2374 & 3.4255 \\
\hline \multirow{2}{*}{5000} & \multirow{2}{*}{5000} & Present & 1.7537 & 1.958 & 3.0634 & 3.9445 & 4.1976 & 5.1453 & 5.2879 \\
& & {$[4]$} & 1.7607 & 1.9901 & 3.1318 & 3.9578 & 4.2553 & 5.2883 & 5.3548 \\
\hline
\end{tabular}

TABLE 8: The first seven frequency parameters $\Omega=\left(\omega b^{2} / \pi^{2}\right)(\rho h / D)^{1 / 2}$ for C-C-F-C Mindlin rectangular plates with translational, rotational, and torsional restraints at $x=a, k_{x a}=K_{x a}=K_{y x a}=K \times D$.

\begin{tabular}{lccccccc}
\hline$K$ & 1 & 2 & 3 & 4 & 5 & 6 & 7 \\
\hline 0 & 2.2388 & 3.6105 & 5.4944 & 6.6135 & 6.8022 & 9.4188 & 9.7667 \\
0.1 & 2.2476 & 3.6233 & 5.5034 & 6.6282 & 6.811 & 9.4295 & 9.775 \\
1 & 2.3132 & 3.7213 & 5.5721 & 6.7447 & 6.8826 & 9.5158 \\
$10^{2}$ & 2.878 & 4.562 & 6.0184 & 7.641 & 7.6707 & 10.2284 & 10.3535 \\
$10^{4}$ & 3.2854 & 6.2286 & 6.2735 & 8.7475 & 10.2438 & 10.4305 \\
$10^{6}$ & 3.2954 & 6.2857 & 6.2861 & 8.8102 & 10.3788 & 10.4778 \\
$10^{7}$ & 3.2955 & 6.2863 & 6.2863 & 8.8109 & 10.3797 & 10.4786 \\
$10^{9}$ & 3.2955 & 6.2863 & 6.2863 & 8.8109 & 10.3797 & 12.3813 \\
$\infty$ & 3.2955 & 6.2863 & 6.2863 & 8.8109 & 10.3797 & 10.4786 \\
\hline
\end{tabular}

\section{Conclusions}

An improved Fourier series method is proposed to analyze the free vibration of Mindlin rectangular plates with general elastic boundary supports. The general boundary conditions are physically realized with the uniform distribution of springs on each boundary edge, and different boundary conditions can be directly obtained by changing the stiffness of the springs. The vibration displacements and the crosssectional rotations of the mid-plane are sought as the linear combination of a double Fourier cosine series and four single auxiliary series functions, respectively. The use of these supplementary functions is to solve the discontinuity problems which were encountered in the displacement and rotations partial differentials along the edges. The unknown expansion coefficients can be solved through using the boundary conditions and the governing equations. In this method, analytical solution is derived for the vibrations of Mindlin rectangular plates with general elastic boundary support. Finally, the numerical results and the comparisons with those reported in the literature are presented to validate the accuracy of the method.

\section{Appendices}

\section{A. Supplementary Series}

We have

$$
\begin{gathered}
\xi_{1 a}(x)=\frac{a}{2 \pi} \sin \frac{\pi x}{2 a}+\frac{a}{2 \pi} \sin \frac{3 \pi x}{2 a}=\sum_{m=0}^{\infty} \alpha_{1 m} \cos \lambda_{m} x, \\
\alpha_{1 m}= \begin{cases}\frac{4 a}{3 \pi^{2}}, & m=0, \\
\frac{2 a}{\left(1-4 m^{2}\right) \pi^{2}}+\frac{6 a}{\left(9-4 m^{2}\right) \pi^{2}}, & m \neq 0,\end{cases}
\end{gathered}
$$




$$
\begin{aligned}
& \xi_{2 a}(x)=-\frac{a}{2 \pi} \cos \frac{\pi x}{2 a}+\frac{a}{2 \pi} \cos \frac{3 \pi x}{2 a}=\sum_{m=0}^{\infty} \alpha_{2 m} \cos \lambda_{m} x, \\
& \xi_{1 a}^{\prime \prime}(x)=-\frac{\pi}{8 a} \sin \frac{\pi x}{2 a}-\frac{9 \pi}{8 a} \sin \frac{3 \pi x}{2 a}=\sum_{m=0}^{\infty} \phi_{1 m} \cos \lambda_{m} x, \\
& \alpha_{2 m}= \begin{cases}-\frac{2 a}{3 \pi^{2}}, & m=0, \\
-\frac{2 a(-1)^{m}}{\left(1-4 m^{2}\right) \pi^{2}}+\frac{6 a(-1)^{m+1}}{\left(9-4 m^{2}\right) \pi^{2}}, & m \neq 0,\end{cases} \\
& \phi_{1 m}= \begin{cases}-\frac{1}{a}, & m=0 \\
-\frac{1}{2\left(1-4 m^{2}\right) a}-\frac{27}{2\left(9-4 m^{2}\right) a}, & m \neq 0\end{cases} \\
& \xi_{1 b}(y)=\frac{b}{2 \pi} \sin \frac{\pi y}{2 b}+\frac{b}{2 \pi} \sin \frac{3 \pi y}{2 b}=\sum_{n=0}^{\infty} \beta_{1 n} \cos \lambda_{n} y, \\
& \xi_{2 a}^{\prime \prime}(x)=\frac{\pi}{8 a} \cos \frac{\pi x}{2 a}-\frac{9 \pi}{8 a} \cos \frac{3 \pi x}{2 a}=\sum_{m=0}^{\infty} \phi_{2 m} \cos \lambda_{m} x, \\
& \beta_{1 n}= \begin{cases}\frac{4 b}{3 \pi^{2}}, & n=0, \\
\frac{2 b}{\left(1-4 n^{2}\right) \pi^{2}}+\frac{6 b}{\left(9-4 n^{2}\right) \pi^{2}}, & n \neq 0,\end{cases} \\
& \xi_{2 b}(y)=-\frac{b}{2 \pi} \cos \frac{\pi y}{2 b}+\frac{b}{2 \pi} \cos \frac{3 \pi y}{2 b}=\sum_{n=0}^{\infty} \beta_{2 n} \cos \lambda_{n} y, \\
& \beta_{2 n}= \begin{cases}-\frac{2 b}{3 \pi^{2}}, & n=0, \\
-\frac{2 b(-1)^{n}}{\left(1-4 n^{2}\right) \pi^{2}}+\frac{6 b(-1)^{n+1}}{\left(9-4 n^{2}\right) \pi^{2}}, & n \neq 0,\end{cases} \\
& \xi_{1 a}^{\prime}(x)=\frac{1}{4} \cos \frac{\pi x}{2 a}+\frac{3}{4} \cos \frac{3 \pi x}{2 a}=\sum_{m=0}^{\infty} \gamma_{1 m} \cos \lambda_{m} x, \\
& \gamma_{1 m}= \begin{cases}\frac{1}{\pi}, & m=0, \\
\frac{(-1)^{m}}{\left(1-4 m^{2}\right) \pi}+\frac{9(-1)^{m+1}}{\left(9-4 m^{2}\right) \pi}, & m \neq 0,\end{cases} \\
& \xi_{2 a}^{\prime}(x)=\frac{1}{4} \sin \frac{\pi x}{2 a}-\frac{3}{4} \sin \frac{3 \pi x}{2 a}=\sum_{m=0}^{\infty} \gamma_{2 m} \cos \lambda_{m} x \\
& \gamma_{2 m}= \begin{cases}0, & m=0, \\
\frac{1}{\left(1-4 m^{2}\right) \pi}-\frac{9}{\left(9-4 m^{2}\right) \pi}, & m \neq 0,\end{cases} \\
& \xi_{1 b}^{\prime}(y)=\frac{1}{4} \cos \frac{\pi y}{2 b}+\frac{3}{4} \cos \frac{3 \pi y}{2 b}=\sum_{n=0}^{\infty} \eta_{1 n} \cos \lambda_{n} y, \\
& \eta_{1 n}= \begin{cases}\frac{1}{\pi}, & n=0, \\
\frac{(-1)^{n}}{\left(1-4 n^{2}\right) \pi}+\frac{9(-1)^{n+1}}{\left(9-4 n^{2}\right) \pi}, & n \neq 0,\end{cases} \\
& \xi_{2 b}^{\prime}(y)=\frac{1}{4} \sin \frac{\pi y}{2 b}-\frac{3}{4} \sin \frac{3 \pi y}{2 b}=\sum_{n=0}^{\infty} \eta_{2 n} \cos \lambda_{n} y, \\
& \eta_{2 n}= \begin{cases}0, & n=0, \\
\frac{1}{\left(1-4 n^{2}\right) \pi}-\frac{9}{\left(9-4 n^{2}\right) \pi}, & n \neq 0,\end{cases} \\
& \phi_{2 m}= \begin{cases}-\frac{1}{2 a}, & m=0 \\
\frac{(-1)^{m}}{2\left(1-4 m^{2}\right) a}-\frac{27(-1)^{m+1}}{2\left(9-4 m^{2}\right) a}, & m \neq 0\end{cases} \\
& \xi_{1 b}^{\prime \prime}(y)=-\frac{\pi}{8 b} \sin \frac{\pi y}{2 b}-\frac{9 \pi}{8 b} \sin \frac{3 \pi y}{2 b}=\sum_{n=0}^{\infty} \varphi_{1 n} \cos \lambda_{n} y, \\
& \varphi_{1 n}= \begin{cases}-\frac{1}{b}, & n=0, \\
-\frac{1}{2\left(1-4 n^{2}\right) b}-\frac{27}{2\left(9-4 n^{2}\right) b}, & n \neq 0,\end{cases} \\
& \xi_{2 b}^{\prime \prime}(y)=\frac{\pi}{8 b} \cos \frac{\pi y}{2 b}-\frac{9 \pi}{8 b} \cos \frac{3 \pi y}{2 b}=\sum_{n=0}^{\infty} \varphi_{2 n} \cos \lambda_{n} y, \\
& \varphi_{2 n}= \begin{cases}-\frac{1}{2 b}, & n=0, \\
\frac{(-1)^{n}}{2\left(1-4 n^{2}\right) b}-\frac{27(-1)^{n+1}}{2\left(9-4 n^{2}\right) b}, & n \neq 0,\end{cases} \\
& \sin \lambda_{m} x=\sum_{p=0}^{\infty} \varepsilon_{m p} \cos \lambda_{p} x \\
& p=0, \quad \varepsilon_{m p}=0, \\
& p \neq 0, \quad \varepsilon_{m p}= \begin{cases}m=0, & \frac{1-(-1)^{p}}{p \pi}, \\
m \neq 0 & \begin{cases}m=p, & 0, \\
m \neq p, & \frac{2 p\left((-1)^{m+p}-1\right)}{\left(m^{2}-p^{2}\right) \pi},\end{cases} \end{cases} \\
& \sin \lambda_{n} y=\sum_{q=0}^{\infty} \varepsilon_{n q} \cos \lambda_{q} y \\
& q=0, \quad \varepsilon_{n q}=0, \\
& q \neq 0, \quad \varepsilon_{n q}= \begin{cases}n=0, & \frac{1-(-1)^{q}}{q \pi} \\
n \neq 0, & \left\{\begin{array}{ll}
n=q, & 0, \\
n \neq q, & \frac{2 q\left((-1)^{n+q}-1\right)}{\left(n^{2}-q^{2}\right) \pi} .
\end{array} .\right.\end{cases}
\end{aligned}
$$




\section{B. Definitions of Matrices}

We have

$$
\begin{aligned}
& \left\{\mathbf{C}_{1,1}\right\}_{s, t}=-\left(\lambda_{m}^{2}+\lambda_{n}^{2}\right) \delta_{s t} \\
& \left\{\mathbf{C}_{1,2}\right\}_{s, t}=-\lambda_{m}^{2} \delta_{s t} \sum_{p=0}^{\infty} \varepsilon_{m p}, \\
& \left\{\mathbf{C}_{1,3}\right\}_{s, t}=-\lambda_{n}^{2} \delta_{s t} \sum_{q=0}^{\infty} \varepsilon_{n q}, \\
& \left\{\mathbf{D}_{1,1}\right\}_{s, m^{\prime}+1}=\left(-\lambda_{m}^{2} \beta_{1 n}+\varphi_{1 n}\right) \delta_{m m^{\prime}}, \\
& \left\{\mathbf{D}_{1,2}\right\}_{s, m^{\prime}+1}=\left(-\lambda_{m}^{2} \beta_{2 n}+\varphi_{2 n}\right) \delta_{m m^{\prime}}, \\
& \left\{\mathbf{D}_{1,3}\right\}_{s, n^{\prime}+1}=\left(-\lambda_{n}^{2} \alpha_{1 m}+\phi_{1 m}\right) \delta_{n n^{\prime}} \\
& \left\{\mathbf{D}_{1,4}\right\}_{s, n^{\prime}+1}=\left(-\lambda_{n}^{2} \alpha_{2 m}+\phi_{2 m}\right) \delta_{n n^{\prime}} \\
& \left\{\mathbf{D}_{1,5}\right\}_{s, m^{\prime}+1}=-\lambda_{m} \beta_{1 n} \delta_{m m^{\prime}} \sum_{p=0}^{\infty} \varepsilon_{m p} \text {, } \\
& \left\{\mathbf{D}_{1,6}\right\}_{s, m^{\prime}+1}=-\lambda_{m} \beta_{2 n} \delta_{m m^{\prime}} \sum_{p=0}^{\infty} \varepsilon_{m p}, \\
& \left\{\mathbf{D}_{1,7}\right\}_{s, n^{\prime}+1}=\gamma_{1 m} \delta_{n n^{\prime}}, \quad\left\{\mathbf{D}_{1,8}\right\}_{s, n^{\prime}+1}=\gamma_{2 m} \delta_{n n^{\prime}}, \\
& \left\{\mathbf{D}_{1,9}\right\}_{s, m^{\prime}+1}=\eta_{1 n} \delta_{m m^{\prime}}, \quad\left\{\mathbf{D}_{1,10}\right\}_{s, m^{\prime}+1}=\eta_{2 n} \delta_{m m^{\prime}} \text {, } \\
& \left\{\mathbf{D}_{1,11}\right\}_{s, n^{\prime}+1}=-\lambda_{n} \alpha_{1 m} \delta_{n n^{\prime}} \sum_{q=0}^{\infty} \tau_{n q}, \\
& \left\{\mathbf{D}_{1,12}\right\}_{s, n^{\prime}+1}=-\lambda_{n} \alpha_{2 m} \delta_{n n^{\prime}} \sum_{q=0}^{\infty} \tau_{n q}, \\
& \left\{\mathbf{E}_{1,1}\right\}_{s, t}=\delta_{s t}, \quad\left\{\mathbf{E}_{1,2}\right\}_{s, t}=0 \text {, } \\
& \left\{\mathbf{E}_{1,3}\right\}_{s, t}=0 \text {, } \\
& \left\{\mathbf{F}_{1,1}\right\}_{s, m^{\prime}+1}=\beta_{1 n} \delta_{m m^{\prime}} \\
& \left\{\mathbf{F}_{1,1}\right\}_{s, m^{\prime}+1}=\beta_{2 n} \delta_{m m^{\prime}} \\
& \left\{\mathbf{F}_{1,3}\right\}_{s, n^{\prime}+1}=\alpha_{1 m} \delta_{n n^{\prime}} \text {, } \\
& \left\{\mathbf{F}_{1,4}\right\}_{s, n^{\prime}+1}=\alpha_{2 m} \delta_{n n^{\prime}}, \\
& \left\{\mathbf{F}_{1,5}\right\}_{s, m^{\prime}+1}=0, \quad\left\{\mathbf{F}_{1,6}\right\}_{s, m^{\prime}+1}=0, \\
& \left\{\mathbf{F}_{1,7}\right\}_{s, n^{\prime}+1}=0, \quad\left\{\mathbf{F}_{1,8}\right\}_{s, n^{\prime}+1}=0, \\
& \left\{\mathbf{F}_{1,9}\right\}_{s, m^{\prime}+1}=0, \quad\left\{\mathbf{F}_{1,10}\right\}_{s, m^{\prime}+1}=0 \text {, } \\
& \left\{\mathbf{F}_{1,11}\right\}_{s, n^{\prime}+1}=0, \quad\left\{\mathbf{F}_{1,12}\right\}_{s, n^{\prime}+1}=0 \text {, } \\
& \left\{\mathbf{H}_{1,1}\right\}_{n+1, m+1}=-k_{x 0} \beta_{1 n}, \\
& \left\{\mathbf{H}_{1,2}\right\}_{n+1, m+1}=-k_{x 0} \beta_{2 n}, \\
& \left\{\mathbf{H}_{1,3}\right\}_{n+1, n^{\prime}+1}=-k c h \delta_{n n^{\prime}},
\end{aligned}
$$

$$
\begin{gathered}
\left\{\mathbf{H}_{1,4}\right\}_{n+1, n^{\prime}+1}=0, \\
\left\{\mathbf{H}_{1,5}\right\}_{n+1, m+1}=-k \operatorname{ch} \beta_{1 n}, \\
\left\{\mathbf{H}_{1,6}\right\}_{n+1, m+1}=-k \operatorname{ch} \beta_{2 n}, \\
\left\{\mathbf{H}_{1,7}\right\}_{n+1, n^{\prime}+1}=0, \quad\left\{\mathbf{H}_{1,8}\right\}_{n+1, n^{\prime}+1}=0, \\
\left\{\mathbf{H}_{1,9}\right\}_{n+1, m+1}=0, \quad\left\{\mathbf{H}_{1,10}\right\}_{n+1, m+1}=0, \\
\left\{\mathbf{H}_{1,11}\right\}_{n+1, n^{\prime}+1}=0, \quad\left\{\mathbf{H}_{1,12}\right\}_{n+1, n^{\prime}+1}=0, \\
\left\{\mathbf{Q}_{1,1}\right\}_{s, t}=k_{x 0} \delta_{m m^{\prime}}, \quad\left\{\mathbf{Q}_{1,2}\right\}_{s, t}=k G h \delta_{m m^{\prime}}, \\
\left\{\mathbf{Q}_{1,3}\right\}_{s, t}=0,
\end{gathered}
$$

where $m^{\prime}=0,1, \ldots, M, m=0,1, \ldots, M, n^{\prime}=0,1, \ldots, N$, $n=0,1, \ldots, N, s=m(N+1)+n+1$, and $t=m^{\prime}(N+1)+n^{\prime}+1$.

\section{Conflict of Interests}

The authors declare that there is no conflict of interests regarding the publication of this paper.

\section{Acknowledgments}

The authors acknowledge the support of this work by the National Natural Science Foundation of China (no. 51105087) and the Fundamental Research Funds for the Central Universities (HEUCF110701). The authors would like to express their sincere thanks to the reviewers for the constructive and positive comments.

\section{References}

[1] K. M. Liew, Y. Xiang, and S. Kitipornchai, “Transverse vibration of thick rectangular plates-I. Comprehensive sets of boundary conditions," Computers and Structures, vol. 49, no. 1, pp. 1-29, 1993.

[2] K. M. Liew, K. C. Hung, and M. K. Lim, "Vibration of mindlin plates using boundary characteristic orthogonal polynomials," Journal of Sound and Vibration, vol. 182, no. 1, pp. 77-90, 1995.

[3] Y. Xiang, K. M. Liew, and S. Kitipornchai, "Vibration analysis of rectangular mindlin plates resting on elastic edge supports," Journal of Sound and Vibration, vol. 204, no. 1, pp. 1-16, 1997.

[4] D. Zhou, "Vibrations of Mindlin rectangular plates with elastically restrained edges using static Timoshenko beam functions with the Rayleigh-Ritz method," International Journal of Solids and Structures, vol. 38, no. 32-33, pp. 5565-5580, 2001.

[5] F.-L. Liu and K. M. Liew, "Analysis of vibrating thick rectangular plates with mixed boundary constraints using differential quadrature element method," Journal of Sound and Vibration, vol. 225, no. 5, pp. 915-934, 1999.

[6] P. Malekzadeh and S. A. Shahpari, "Free vibration analysis of variable thickness thin and moderately thick plates with elastically restrained edges by DQM," Thin-Walled Structures, vol. 43, no. 7, pp. 1037-1050, 2005.

[7] Y. Hou, G. W. Wei, and Y. Xiang, "DSC-Ritz method for the free vibration analysis of Mindlin plates," International Journal for Numerical Methods in Engineering, vol. 62, no. 2, pp. 262-288, 2005. 
[8] Y. Xiang, S. K. Lai, and L. Zhou, "DSC-element method for free vibration analysis of rectangular Mindlin plates," International Journal of Mechanical Sciences, vol. 52, no. 4, pp. 548-560, 2010.

[9] S. H. Hashemi and M. Arsanjani, "Exact characteristic equations for some of classical boundary conditions of vibrating moderately thick rectangular plates," International Journal of Solids and Structures, vol. 42, no. 3-4, pp. 819-853, 2005.

[10] S. H. Hashemi, K. Khorshidi, and H. Rokni Damavandi Taher, "Exact acoustical analysis of vibrating rectangular plates with two opposite edges simply supported via Mindlin plate theory," Journal of Sound and Vibration, vol. 322, no. 4-5, pp. 883-900, 2009.

[11] D. J. Gorman, "Free vibration analysis of Mindlin plates with uniform elastic edge support by the superposition method," Journal of Sound and Vibration, vol. 207, no. 3, pp. 335-350, 1997.

[12] F. Ohya, M. Ueda, T. Uchiyama, and M. Kikuchi, "Free vibration analysis by the superposition method of rectangular Mindlin plates with internal columns resting on uniform elastic edge supports," Journal of Sound and Vibration, vol. 289, no. 1-2, pp. $1-24,2006$.

[13] W. L. Li, "Free vibrations of beams with general boundary conditions," Journal of Sound and Vibration, vol. 237, no. 4, pp. 709-725, 2000.

[14] W. L. Li, "Vibration analysis of rectangular plates with general elastic boundary supports," Journal of Sound and Vibration, vol. 273, no. 3, pp. 619-635, 2004.

[15] J. Du, W. L. Li, G. Jin, T. Yang, and Z. Liu, "An analytical method for the in-plane vibration analysis of rectangular plates with elastically restrained edges," Journal of Sound and Vibration, vol. 306, no. 3-5, pp. 908-927, 2007.

[16] W. L. Li, X. Zhang, J. Du, and Z. Liu, "An exact series solution for the transverse vibration of rectangular plates with general elastic boundary supports," Journal of Sound and Vibration, vol. 321, no. 1-2, pp. 254-269, 2009. 

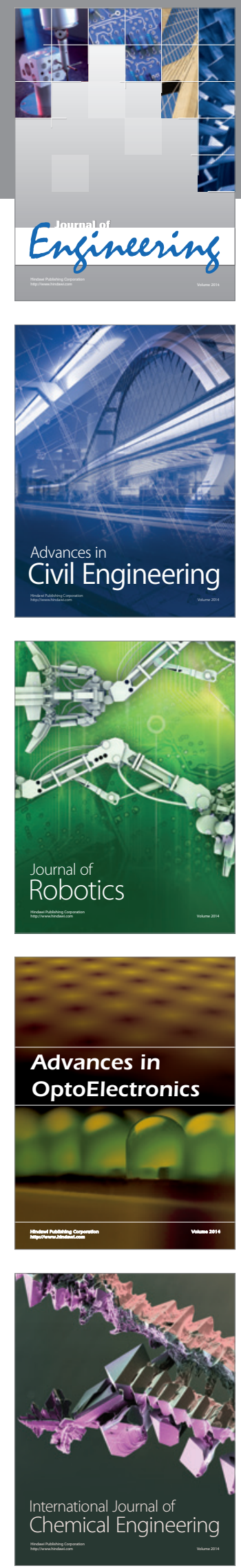

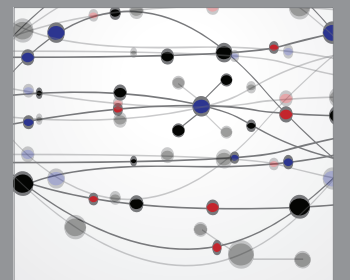

The Scientific World Journal
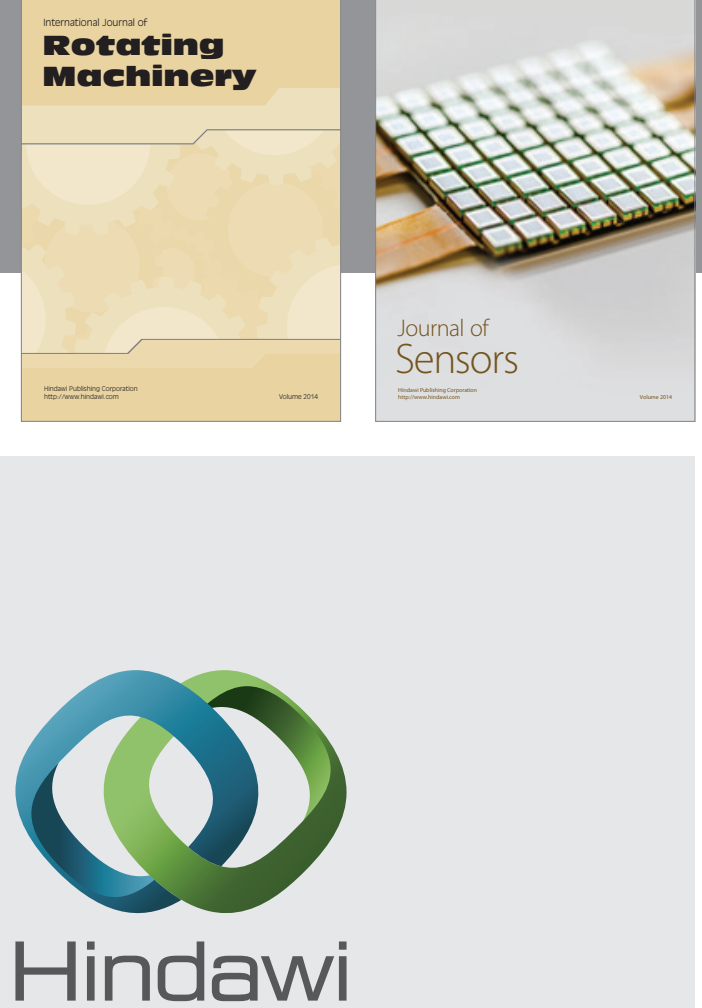

Submit your manuscripts at http://www.hindawi.com
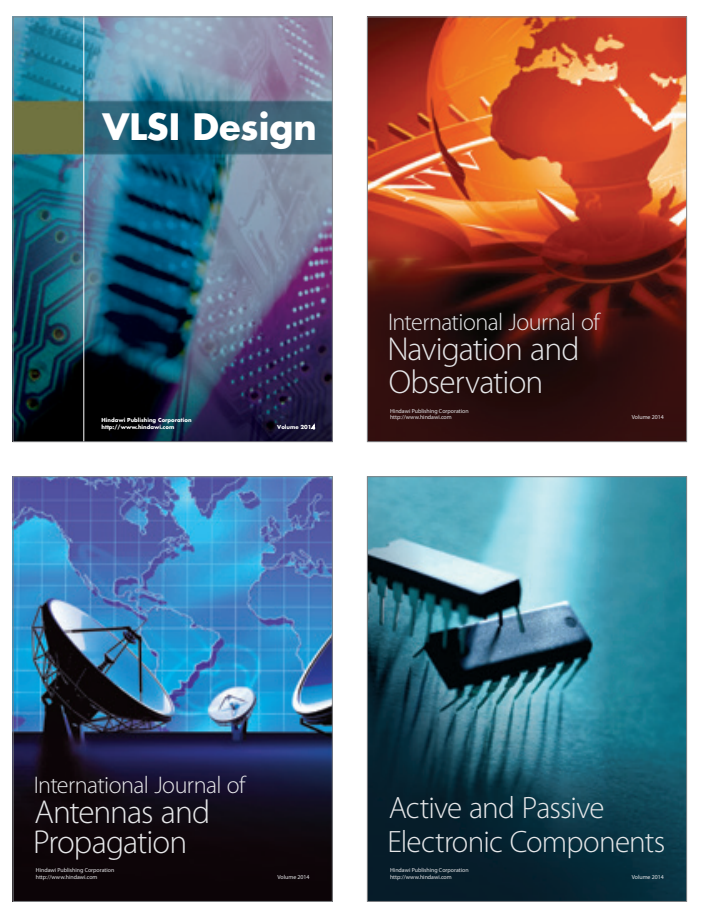
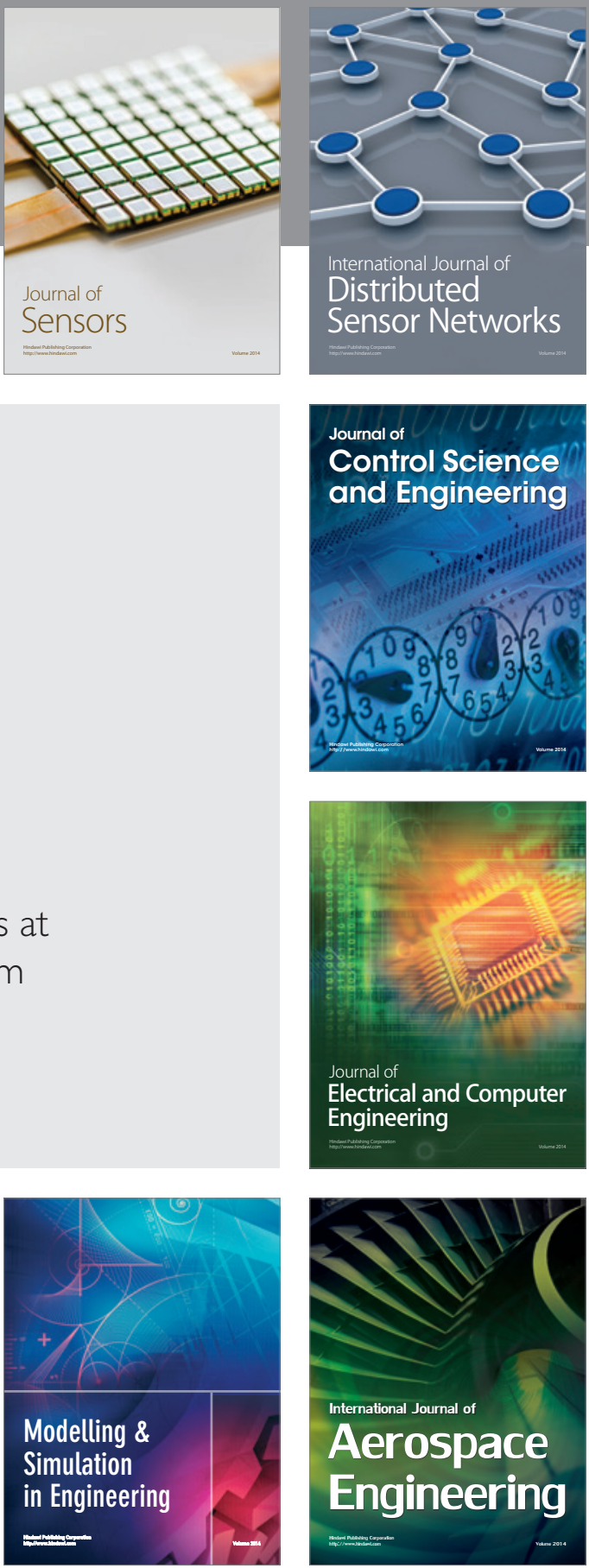

Journal of

Control Science

and Engineering
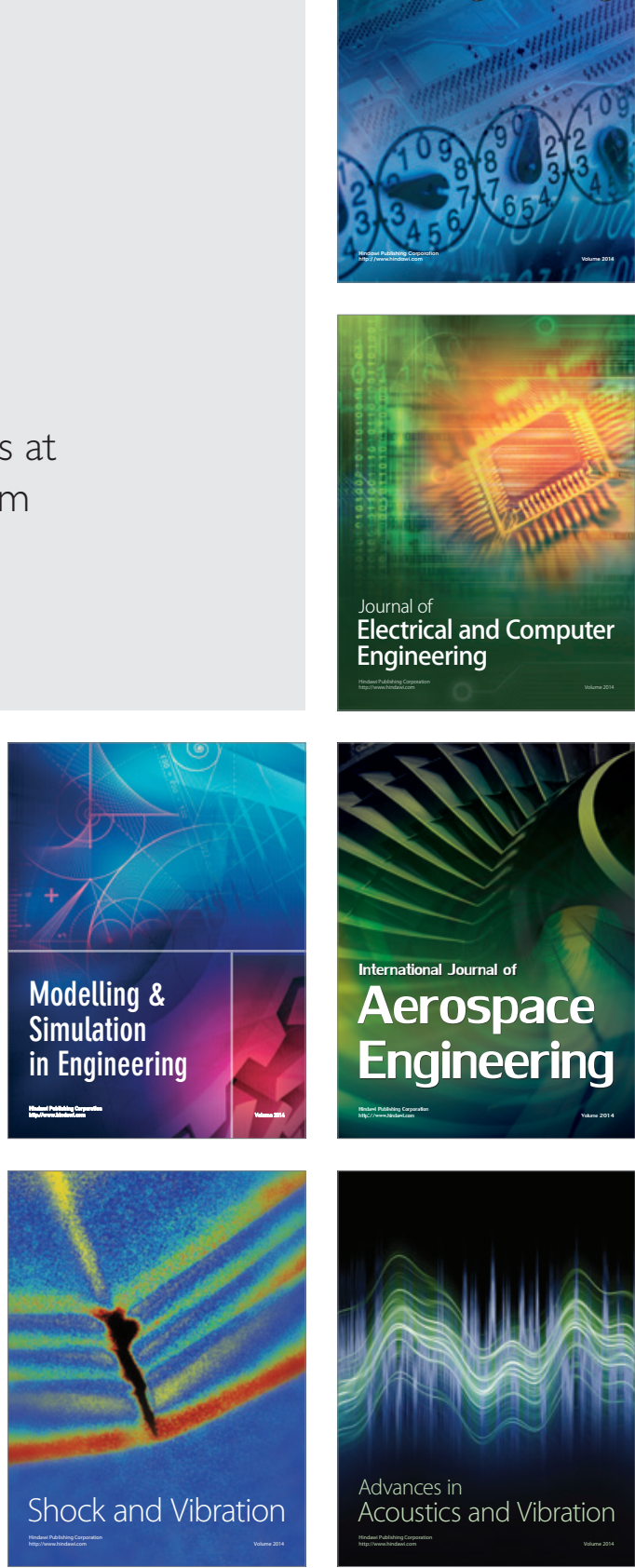